\title{
Negative surgical exploration in suspected gastrointestinal perforation: trend, preoperative predictors, and etiologies
}

\author{
Xuan Liu, Weizhong Sheng, Yuda Gong, Weidong Gao, Bo Zhang \\ Department of General Surgery, Zhongshan Hospital, Fudan University School of Medicine, Shanghai, China \\ Contributions: (I) Conception and design: X Liu, B Zhang; (II) Administrative support: W Gao; (III) Provision of study materials or patients: X Liu, \\ W Sheng, Y Gong; (IV) Collection and assembly of data: X Liu, W Sheng, Y Gong; (V) Data analysis and interpretation: X Liu, Y Gong; (VI) \\ Manuscript writing: All authors; (VII) Final approval of manuscript: All authors. \\ Correspondence to: Bo Zhang. Department of General Surgery, Zhongshan Hospital, Fudan University School of Medicine, No. 180 Fenglin Road, \\ Shanghai 200032, China. Email: zhang.bo@zs-hospital.sh.cn.
}

Background: Despite the rapid improvement of clinical science and imaging technology including
computed tomography, the entity of negative surgical exploration in suspected gastrointestinal perforation
(N-GIP) still exist. However, few studies have focused on this issue and most studies are case reports. We
undertook this study to investigate the rates of N-GIP, and explore a set of possible preoperative predictors
associated with N-GIP.

Methods: This was a retrospective study performed at the department of general surgery in our treatment center. All patients included were suspected gastrointestinal perforation (GIP) cases, aged 14 years and over, and underwent emergency surgery between 2009 and 2019. A predictive multivariable model of the presence of N-GIP was developed using logistic regression analysis.

Results: A total of 973 patients were identified and 30 (3.1\%) were found to have no evidence of perforated gastrointestinal tract. The mean age of patients was 59.74 (range, 14-97) years, and 67.2 percent of the patients were males. The rates of N-GIP did not have a significant change over time $(\mathrm{P}=0.212$ for trend). In multivariable analysis, absence of generalized peritonitis, duration of abdominal pain $>19.6$ hours, and neutrophil-to-lymphocyte ratio (NLR) $<3.80$ were significant predictors of N-GIP. N-GIP was more common in patients with gastrointestinal tumors and foreign bodies. Five patients $(16.7 \%)$ in N-GIP group experienced complications and the 90 -day mortality rate was $6.7 \%$.

Conclusions: The rates of N-GIP did not change significantly over time. N-GIP was associated with the absence of generalized peritonitis, duration of abdominal pain $>19.6$ hours, and NLR $<3.80$.

Keywords: Gastrointestinal perforation (GIP); emergency laparotomy; negative laparotomy; preoperative predictors

Submitted Dec 24, 2020. Accepted for publication Mar 21, 2021.

doi: 10.21037/atm-20-8158

View this article at: http://dx.doi.org/10.21037/atm-20-8158

\section{Introduction}

Gastrointestinal perforation (GIP) is defined as the destruction of integrity of the gastrointestinal tract. GIP usually presents as an acute abdominal condition, with localized or generalized peritonitis. A large percentage of all hospital admissions for emergency-surgery in General Surgery Department are secondary to perforation of abdominal viscera (1). GIP is a surgical emergency that carries a high risk for mortality (30-50\%) despite improvements in antibiotics, radiographic imaging, resuscitation therapy, and surgical technique $(2,3)$. Many individuals who suffer from GIP develop SIRS or severe sepsis accompanied by hemodynamic compromise, coagulopathy, acidosis, and hypothermia $(4,5)$. As a lifethreatening disease (especially in elderly patients), GIP 
usually needs emergency surgery and active rescue in surgical ICU. GIP can occur secondary to various conditions such as iatrogenic and traumatic causes, neoplasms, inflammation, or peptic ulcers. In general, many factors may contribute to GIP, including older age, diabetes, glucocorticoid therapy, antecedent diverticulitis, and usage of bevacizumab, tofacitinib, tocilizumab, nonsteroidal anti-inflammatory drugs (NSAIDs), or other biologic agents (6-8). Successful treatment requires a complete understanding of the pathophysiology, anatomy, and microbiology of the disease process and thorough knowledge of the therapy, such as antibiotics, source control, physiologic support and resuscitation (2).

Operative management for patients clinically suspected of having GIP are the most effective and important treatment methods $(9,10)$. However, such procedures are rarely performed in the absence of perforation upon macroscopic or microscopic examination, a circumstance called as negative surgical exploration in suspected of GIP (N-GIP), which means we had no significant findings during a surgical exploration in patients who were clinically suspected of having GIP. N-GIP may lead to a palpable degree of morbidity and mortality, an appreciable prolongation of hospital stay, and complications because of postoperative infection. It is also a main contributor to medical conflict and healthcare costs.

Radiographic evaluation of GIP traditionally begins with upright and supine abdominal radiographs. Currently, helical CT has become a preferred tool to assess patients suspected of having GIP (11). Oral or intravenous contrast agents are used to facilitate identification of perforation when feasible. Accurate and confirmative preoperative diagnosis of GIP remains challenging despite proper history taking, clinical examination, and improvement in new imaging techniques like ultrasound imaging and computed tomography. Preoperative diagnosis of GIP is very crucial to minimize the morbidity and mortality in densely populated countries like China. As management errors can be significantly reduced by correct preoperative diagnosis, clinical acumen plays a pivotal role in the diagnosis and management of GIP and ensures definitive per-operative surgical treatment, which in turn minimizes negative laparotomy or laparoscopy. Thus, surgeons need to build up diagnostic acumen and decisionmaking in the management of GIP.

Regardless of the development of evidence-based guidelines and medical technology, it remains unclear whether a reduction in rates of N-GIP has been achieved over time. Until recently, far too little attention has been drawn to this issue and the overall problem of N-GIP has remained refractory. The aim of the present study was thus to analyze the characterization of its prevalence and clinical predictors, summarize the causes of N-GIP so as to provide objective basis for surgeons' decision-making. We present the following article in accordance with the STROBE reporting checklist (available at http://dx.doi.org/10.21037/ atm-20-8158).

\section{Methods}

We performed a retrospective analysis of clinical suspected GIP cases that underwent an emergency surgery on the same admission to Zhongshan Hospital of Fudan University between 2009 and 2019. Patients who either had an elective surgical exploration or were younger than 14 years of age at the time of operation were excluded from this study.

The preoperative data recorded were as follows: demographic information (age and sex), clinical symptoms at presentation (abdominal pain) and clinical signs (sign of peritonitis), duration of abdominal pain, reports from abdominal US, abdominal radiographs, CT, MRI, endoscope. The white blood cell count (WBC), percentage of neutrophils, percentage of lymphocytes, neutrophilto-lymphocyte ratio (NLR), C-reactive protein (CRP), procalcitonin (PCT), D-dimer, and preoperative vital signs (temperature, shock), ASA grades were also collected. Operative factors such as surgical approach and operative findings were obtained as well by reviewing the operative notes. Postoperative data included any complications recorded within the 90-day postoperative period and histopathological findings.

The classification of signs of peritonitis was based on the results of physical examinations by which surgeons determined whether there were signs of peritonitis and whether the clinical signs of peritonitis were localized or generalized. Considering laboratory results and vital signs fluctuating of patients during the course of medical attention, we used the highest recorded value before surgical exploration for our analysis, or "peak preoperative" value, rather than using presented values at certain times.

In this study, N-GIP was defined as the finding of a macroscopically and/or microscopically scrutinized complete gastrointestinal tract without gastrointestinal wall defect in a patient who underwent emergency operation due to preoperative clinical diagnosis of GIP determined by general surgeons based on a set of evidences including medical history, physical examination, laboratory results, 
imaging studies and diagnostic abdominal paracentesis.

\section{Statistical analysis}

Descriptive statistics are reported as means and standard deviation, median with interquartile range, or as otherwise indicated. Differences in continuous and categorical data between groups were analyzed by means of Student's $t$-test, Chi-square test and Mann-Whitney U-test respectively. Rates of outcomes were tested for changes over time using $\chi^{2}$ test for trend.

Multivariable model predicting the presence of N-GIP was performed with demographic and clinical factors found to be statistically significant in univariable analysis in a machine learning model by gradient boosting decision tree model (catboost) from our model development cohort ( $70 \%$ of patients). A validation set of the remaining $30 \%$ of patients was built to test the model. After the model training was completed, the SHAP value of each feature was calculated to reflect the importance of the feature, and the relationship between the feature and the outcome variable was explored. The SHAP value can guide the grouping of continuous variables, which can be incorporated into the logistic regression model.

The predictors of N-GIP were identified using a binary logistic regression modeling technique. Odds ratios (OR) and $95 \%$ confidence intervals (CI) were determined in the regression models. Overall model fit was assessed by estimating the overall likelihood ratio $\chi^{2}$, the HosmerLemeshow goodness-of-fit test, and the C-statistic to measure the discrimination of the model.

A significance level of $\alpha=0.05$ was used for all tests. All $\mathrm{P}$ values were two-sided. All demographic and clinical data of each patient were analyzed by SPSS, version 20.0 for Windows (IBM, Analytics, Armonk, NY, United States) and Python programming language, version 3.6 (Python Software Foundation, https://www.python.org/).

The study was conducted in accordance with the Declaration of Helsinki (as revised in 2013). The study was approved by ethics board of Zhongshan hospital, Fudan university (No.: B2021-116) and individual consent for this retrospective analysis was waived.

\section{Results}

\section{Patients and population}

From 2009 to 2019, 973 patients were admitted to our department with a preoperative diagnosis of GIP. The mean age at the time of the procedure was 59.74 (range, 14-97) years and 67.2 percent of the patients were males. Median ASA grade was II (range, I-IV). In all patients, GIP or N-GIP was finally proved by operative and/ or histopathological findings. All patients suffered from abdominal pain. Most of the patients (91.6\%) were treated by traditional open surgery. Of the patients included, 30 $(3.1 \%)$ were found to have N-GIP.

\section{Rate and position of N-GIP}

During 2009-2019, the number of GIP cases showed an upward trend with an increase of N-GIP cases in recent five years. The rate of $\mathrm{N}-\mathrm{GIP}$ changed insignificantly over time, from 0 percent in 2009 to 2.86 percent in 2019 ( $\mathrm{P}=0.212$, Chi-square test for trend) (Figure 1).

Locations of GIP and sites of N-GIP revealed during surgery and/or in histopathological findings are displayed in Figure 2. N-GIP appeared to occur more frequently in duodenum and right-sided colon.

\section{Preoperative data}

Of the 973 patients, 898 (92.3\%) patients underwent CT scanning and 68 patients underwent abdominal radiographs (among them 16 patients also underwent CT scanning) during the initial evaluation before surgery, and pneumoperitoneum was found in 890 patients. Other examinations in some patients including ultrasonography, MRI, diagnostic abdominal puncture and findings in endoscope were similarly helpful to diagnosis preoperationally. According to the outcome, the sensitivity of CT and abdominal radiographs were $95.6 \%$ and $92.3 \%$. But in 29 patients with N-GIP who underwent CT scanning, only one patient had surely no indications of GIP on CT images while others all had a pneumoperitoneum, focal wall defect or bowel wall thickening.

As CRP, PCT and D-dimer were applied universally and normally in clinical work in recent years, missing data does exist in patients admitted at earlier time points in the observation period, significant rates of missing data were $18.3 \%, 48.1 \%, 31.6 \%$ respectively. These three variables were excluded from the analysis. Table 1 details the demographic and baseline clinical data of the 973 patients who met the inclusion criteria for our study. Percentage of neutrophils, NLR were frequently decreased and signs of peritonitis were less serious for patients with 

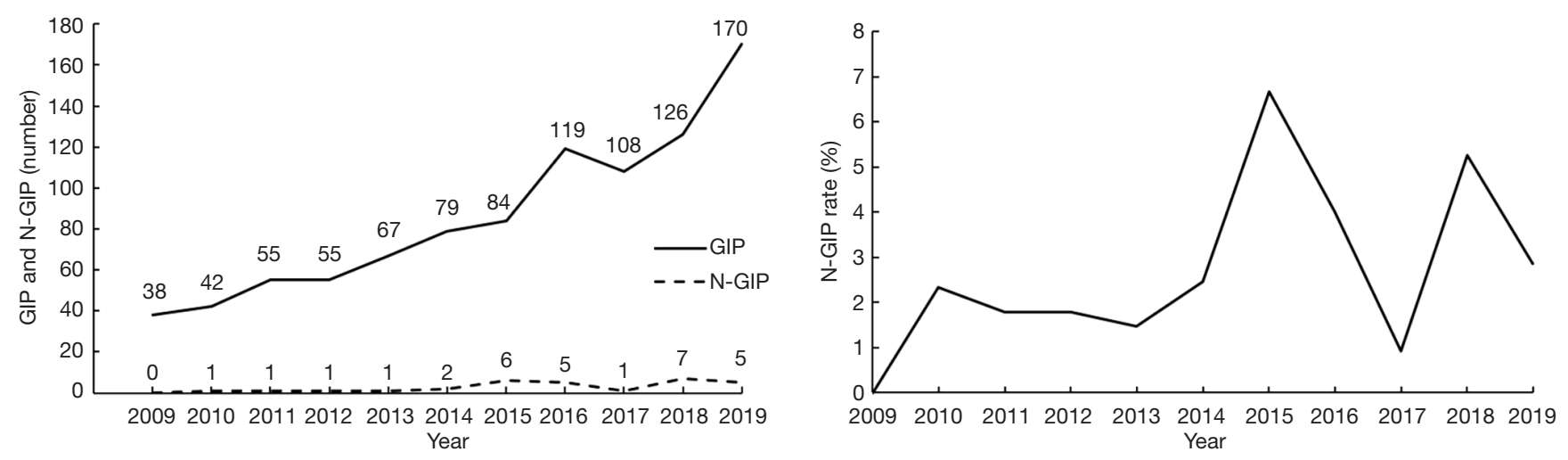

Figure 1 Numbers of GIP and N-GIP cases and rates of N-GIP, 2009-2019. GIP, gastrointestinal perforation; N-GIP, negative surgical exploration in suspected gastrointestinal perforation.

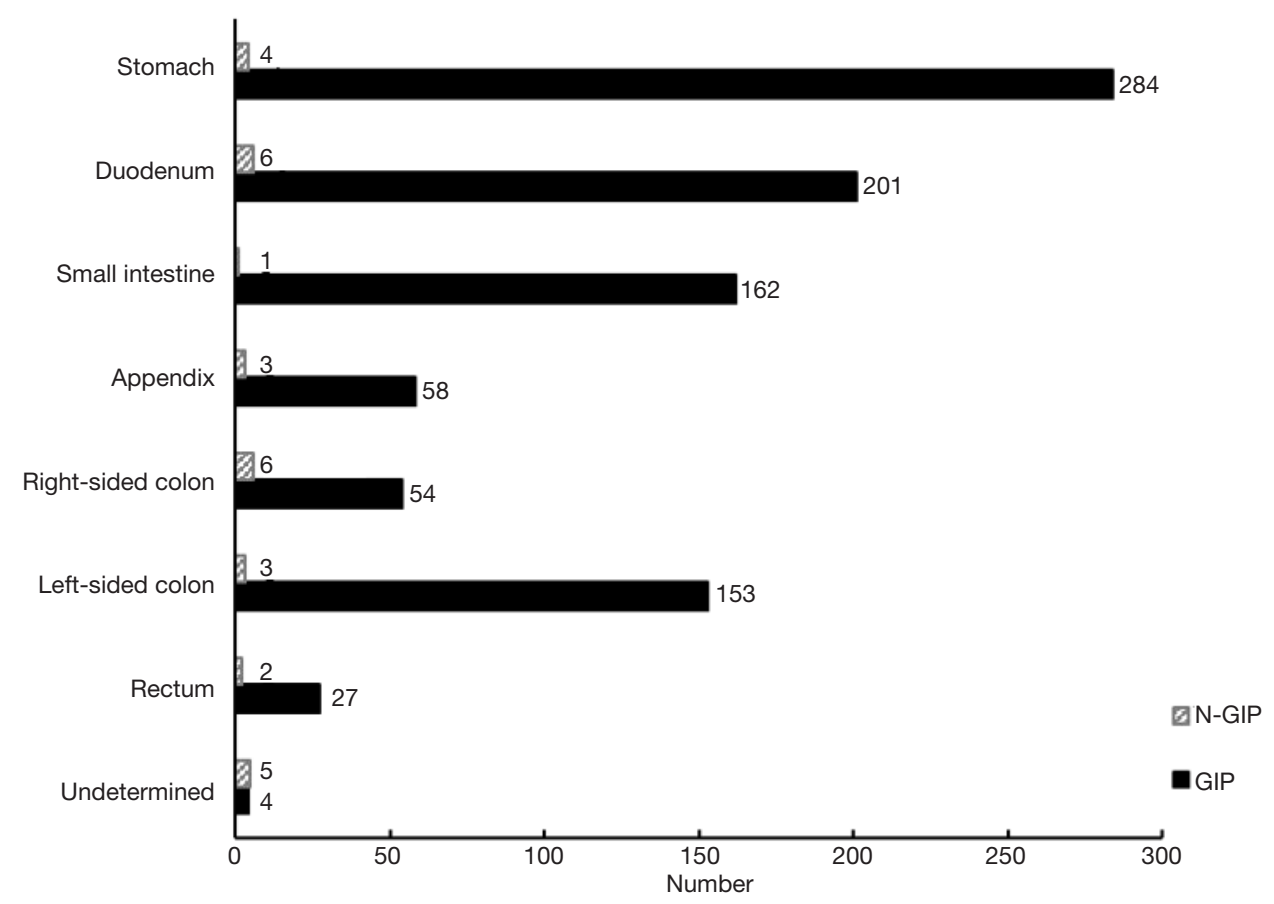

Figure 2 The locations of GIP and sites where N-GIP takes place. Undetermined locations in cases of GIP: due to the special intraoperative conditions, no further exploration is possible to identify the perforation location though gastrointestinal contents were found in abdomen. Undetermined sites in cases of N-GIP: there were no clear indications of gastrointestinal abnormalities with repeating scrutinization. GIP, gastrointestinal perforation; N-GIP, negative surgical exploration in suspected gastrointestinal perforation.

N-GIP. The N-GIP group had statistically significant higher percentage of lymphocytes and longer duration of abdominal pain than patients with GIP. We found no statistically significant differences between the N-GIP and GIP groups for age, sex, ASA grade, shock, temperature and white WBC.

\section{Multivariable predictors for N-GIP}

Training dataset included 681 patients (22 negative) and test dataset included 292 patients ( 8 negative). Because the proportion of negative samples is too low, the model training was weighted according to the ratio of positive: 
Table 1 The characteristics of 973 patients with GIP and N-GIP

\begin{tabular}{|c|c|c|c|}
\hline Characteristics & GIP $(n=943)$ & N-GIP $(n=30)$ & $\mathrm{P}$ \\
\hline Male sex [n (\%)] & $633(67.1)$ & $21(70.0)$ & $0.741^{*}$ \\
\hline ASA grade [n (\%)] & & & $0.539^{\star}$ \\
\hline 1 & $408(43.3)$ & $16(53.3)$ & \\
\hline III & $60(6.4)$ & $2(6.7)$ & \\
\hline IV & $104(11.1)$ & $1(3.3)$ & \\
\hline Duration of abdominal pain [median (IQR)] & $17.0(7.90-48.90)$ & $48.0(20.75-120.00)$ & $<0.001^{\star *}$ \\
\hline Peritonitis [n (\%)] & & & $<0.001^{*}$ \\
\hline Generalized peritonitis & $669(70.9)$ & $4(13.3)$ & \\
\hline Shock [n (\%)] & $124(13.1)$ & $1(3.3)$ & $0.192^{*}$ \\
\hline Temperature & $37.43(0.84)$ & $37.41(0.78)$ & 0.918 \\
\hline WBC & $12.96(6.22)$ & $12.96(5.59)$ & 1.000 \\
\hline Percentage of neutrophils & $85.35(8.90)$ & $81.35(9.24)$ & 0.016 \\
\hline Percentage of lymphocytes & $9.37(7.67)$ & $12.23(7.57)$ & 0.044 \\
\hline NLR & $16.11(13.92)$ & 11.07 (10.09) & 0.049 \\
\hline
\end{tabular}

Values are means and values in parentheses are standard deviations unless indicated otherwise. N (\%), number (percentage). P, Student's t-test. $\mathrm{P}^{\star}$, Chi-square test. $\mathrm{P}^{\star \star}$, Mann-Whitney U-test. GIP, gastrointestinal perforation; N-GIP, negative surgical exploration in suspected gastrointestinal perforation; IQR, interquartile range; ASA, American Society of Anesthesiologists; WBC, white blood cell count; NLR, neutrophil-to-lymphocyte ratio.

negative $=1: 12$ (i.e., the harm of misjudging one negative sample as positive $=$ the harm of misjudging 12 positive samples as negative), which improves the detection of negative samples. In the test dataset, 284 positive patients were detected $(100 \%)$, and 3 of 8 negative patients were detected. Figure 3 shows the relationship between the value of each feature (blue is low, red is high) and the outcome variable. The importance of the features is ranked from high to low. Signs of peritonitis is the most important, and sex is the least important.

By calculating the Shap value of each continuous variable exceeding 0 point (Figure 4), the risk interval of each variable can be calculated as follows: duration of abdominal pain: $>19.6$ hours, percentage of neutrophils: $<68.89$, percentage of lymphocytes: $>6.48$, NLR: $<3.80$. These risk intervals can further guide the grouping of continuous variables, so as to convert continuous variables into categorical variables, and then perform logistic regression to calculate the OR value.

Absence of generalized peritonitis, duration of abdominal pain $>19.6$ hours, and NLR $<3.80$ were all significant predictors of N-GIP (Table 2). The multivariable models demonstrated good model fit and high discrimination (likelihood ratio $\chi^{2}=95.31, \mathrm{P}<0.001$; the Hosmer-Lemeshow goodness-of-fit test $\mathrm{P}=0.964)$. ROC curve showed that the area under the ROC curve of the preoperative multivariable model to distinguish GIP or N-GIP is 0.90 .

\section{Emergency surgical procedures and postoperative outcome}

Of the 30 patients with N-GIP, $15(50.0 \%)$ had a therapeutic operation with the finding of intra-abdominal diseases which required management, but only 9 needed an emergency surgical treatment, such as removal of the 


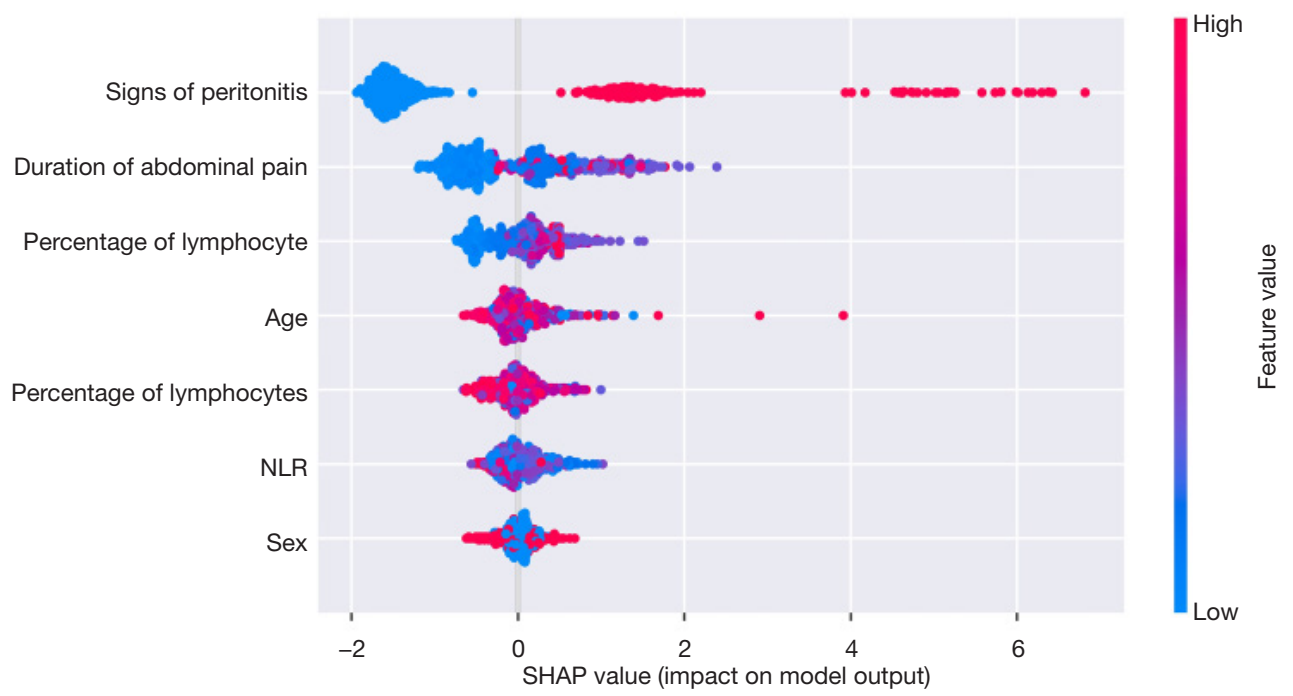

Figure 3 The relationship between the value of each feature and the outcome variable. The abscissa represents the contribution of the feature to the outcome variable: less than 0 represents a protective effect, and greater than 0 represents a dangerous effect. NLR, neutrophilto-lymphocyte ratio.
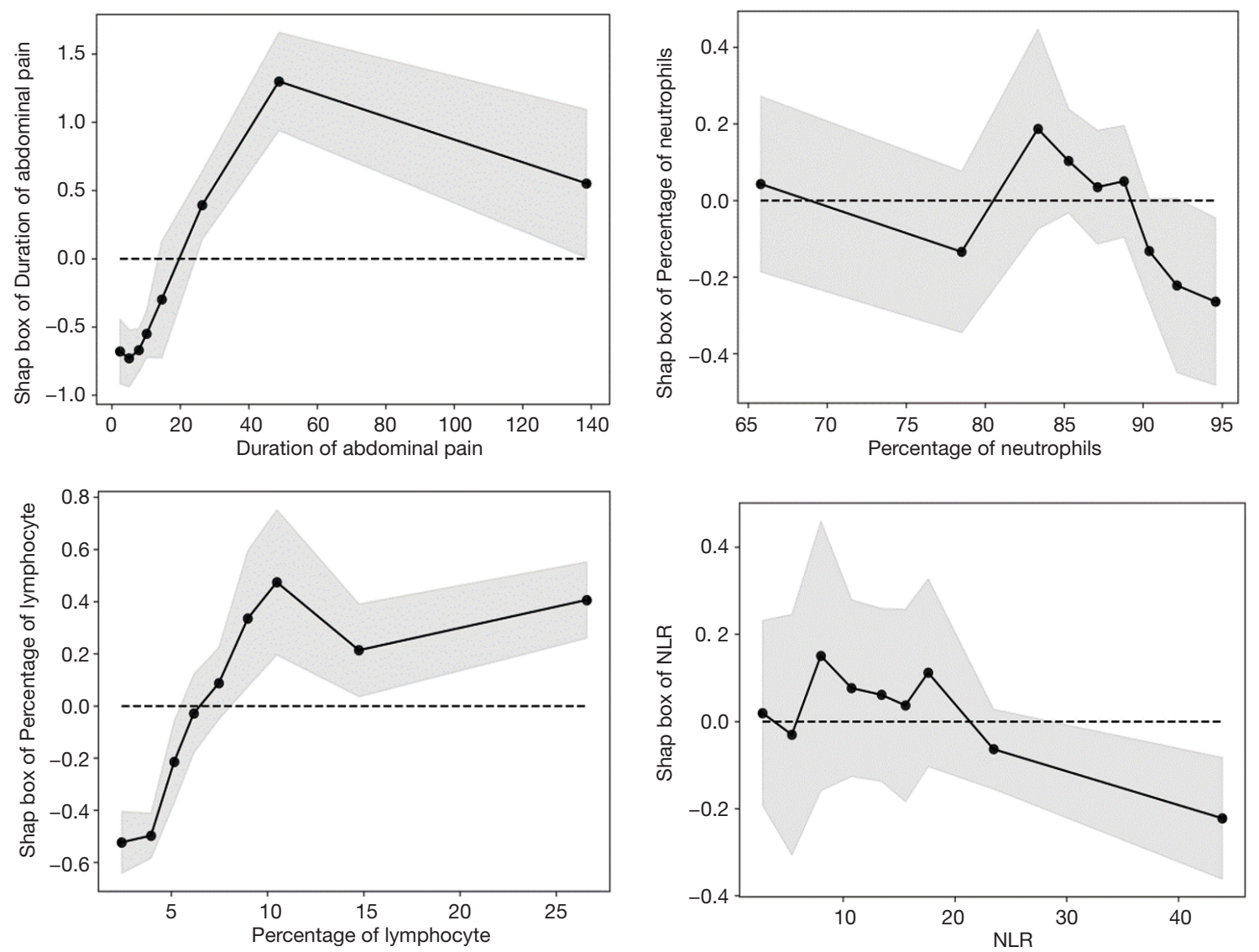

Figure 4 The shap box of duration of abdominal pain, neutrophils, lymphocytes and NLR. NLR, neutrophil-to-lymphocyte ratio. 
Table 2 Predictors and multivariable logistic regression model predicting N-GIP (model with significant terms only)

\begin{tabular}{|c|c|c|c|}
\hline Predictors & \multicolumn{3}{|c|}{ Multivariable logistic regression } \\
\hline Duration of abdominal pain $>19.6 \mathrm{~h}$ & 3.39 & 0.024 & $1.17-9.80$ \\
\hline Sign of peritonitis (versus generalized peritonitis) & & $<0.001$ & \\
\hline Localized peritonitis & 4.9 & 0.009 & $1.49-16.09$ \\
\hline$N L R<3.80$ & 2.9 & 0.019 & $1.19-7.10$ \\
\hline
\end{tabular}

N-GIP, negative surgical exploration in suspected gastrointestinal perforation; NLR, neutrophil-to-lymphocyte ratio.

Table 3 Causes of N-GIP

\begin{tabular}{lcc}
\hline Etiologies of N-GIP & Number & Percentage (\%) \\
\hline Tumor & 7 & 23.3 \\
Foreign body & 5 & 16.7 \\
Appendicitis & 3 & 10.0 \\
Gastrointestinal wall inflammation & 3 & 10.0 \\
Spontaneously sealed small & 3 & 10.0 \\
perforation & & \\
Primary peritonitis & 2 & 6.7 \\
Trauma & 2 & 6.7 \\
Therapeutic colonoscopy & 2 & 6.7 \\
Peptic ulcer & 1 & 3.3 \\
Diverticulitis & 1 & 3.3 \\
Abdominal hematoma & 1 & 3.3 \\
\hline N-GIP, negative surgical exploration in suspected gastrointestinal \\
perforation.
\end{tabular}

foreign bodies from gastrointestinal tract. Four patients lacked the indications for emergency surgical treatment and could be managed by selective operation after perfect preoperative preparation. Two patients underwent a gastrostomy or enterostomy because of unresectable advanced tumor. The remaining 15 patients $(50.0 \%)$ had a non-therapeutic laparotomy $(n=10)$, laparoscopy $(n=3)$ or conversion to laparotomy $(\mathrm{n}=2)$.

Interestingly, the N-GIP group did not have a significantly lower rate of complications and mortality than the GIP group ( $\mathrm{P}=0.131$ and 1$)$. Five of the 30 patients (16.7\%) with N-GIP and 277 of the 943 patients (29.4\%) with GIP had complications. The 90 -day mortality in the N-GIP group and GIP group was $6.7 \%$ and $6.5 \%$, respectively.

\section{Etiology of N-GIP}

We thoroughly reviewed the whole process of disease progression, treatment and postoperative recovery, searched and studied similar case series and eventually identify the causes of N-GIP (Table 3). N-GIP were most often due to tumor and foreign body of the gastrointestinal tract.

\section{Discussion}

Precise diagnosis of GIP is still challenging despite improvement in clinical examinations and investigative procedures. If the preoperative diagnosis is indecisive it is suggested by clinical experience to operate for exploration immediately rather than waiting and observation. However, such a strategy will certainly raise the unnecessary surgical exploration rate and the morbidity. Most of the previous researches merely focused on reviewing the causes of nonsurgical pneumoperitoneum and numerous studies were case reports (12-17).

As early as 1983, Roh and his colleagues (18) reported that 14 percent of patients in their study who had abdominal pain and pneumoperitoneum on plain abdominal radiographs underwent an unnecessary laparotomy owing to the fact that pneumoperitoneum was from sources other than a perforated hollow viscus. They believed that the clinical manifestations of their patients who had surgical versus nonsurgical causes of pneumoperitoneum was indistinguishable. In reality, pneumoperitoneum reflects perforation of abdominal viscus in $85 \%$ to $95 \%$ of total occurrences. Whereas in $5 \%$ to $15 \%$ of cases, pneumoperitoneum does not reflect visceral perforation and arises from another source that does not demand 
emergency surgery (19). Nonsurgical pneumoperitoneum is defined by the existence of air in the peritoneal space that is detectable by roentgenogram and either is treated successfully by observation and supportive care alone or leads to a nondiagnostic laparotomy (20). In this study, the rate of nonsurgical pneumoperitoneum was $2.9 \%$. Case reports and small series (21-24) have established the entity of nonsurgical pneumoperitoneum and the corresponding indication for conservative treatment in selected cases.

This analysis of 973 patients undergoing surgical exploration for suspected GIP provides compelling evidence. The overall rate of N-GIP at our hospital has not decreased over time with the development of evidencebased guidelines and medical technology. N-GIP cases have been encountered more frequently in clinical work in recent five years and caused a myriad of unexpected troubles. Since this study and other studies $(11,25,26)$ had reported such high diagnostic sensitivity of CT or KUB, it is crucial to pay attention to the issue of misdiagnosis and mistreatment.

It is a good choice to consider some clinical features when selecting appropriate surgical candidates in patients with suspected GIP. Our findings regard that the association of signs of peritonitis, duration of abdominal pain, percentage of neutrophils, percentage of lymphocytes, NLR, and negative laparotomy were notable. These associations indicated that the clinical characteristics of patients with GIP versus patients with N-GIP may be different. Patients with N-GIP had a slow process of the disease, minimal signs of peritonitis, and lower level of inflammatory indexes due to no destruction of integrity of the gastrointestinal tract and no gastrointestinal contents in the abdominal cavity. Absence of generalized peritonitis, duration of abdominal pain $>19.6$ hours, and NLR $<3.80$ were identified as significant predictors of N-GIP in the final analyses. The most common sites of N-GIP were duodenum and rightsided colon. Gastrointestinal cancer was the commonest cause of N-GIP in this study, which comprised $23.3 \%$ of the total patients. Foreign body in gastrointestinal tract accounted for $16.7 \%$ of the total patients. Appendicitis, gastrointestinal inflammation and spontaneously sealed small perforation respectively constituted $10 \%$ of the causes. Therefore, we suggest that when duration of abdominal pain is long-lasting, generalized peritonitis is absent, and NLR is in a lower level, the possibility of negative surgical exploration should be considered in preoperative assessment for patients suspected GIP, especially in patients with gastrointestinal cancer. We should implement subsequent investigation with additional preoperative diagnostics for selected patients with risk factors for N-GIP, for example, repeated paracentesis judging for presence of GIP. From our perspective, it is indispensable to perform careful and thorough history and physical examination, and to make judgments based on clinical symptoms and signs, laboratory values, imaging and other examinations.

Unexpectedly, our study demonstrated that there were nearly no differences in the rate of complications and mortality between patients who had GIP versus N-GIP. Five patients $(16.7 \%)$ in the 30 patients with negative finding in operation experienced a complication and two in them later succumbed to postoperative complications. Four patients in N-GIP group underwent laparoscopic surgery, all had no complications in postoperative period. With the rapid development of minimally invasive concept and techniques in recent years, the application of laparoscopy in the diagnosis and treatment of patients with GIP has become more frequent (27-29). However, there are few high-quality clinical studies and many researches are biased. When the difficulty of the clinical decision making for patients suspected of having GIP is unsolved, laparoscopic exploration may be a simply good management and of great help to surgeons.

A major limitation of this study is that it was retrospective and patients were selected from a single center, which made the evidence level for this study relatively low. The event rate of only 30 patients with N-GIP over a 11-year period is low and reflective of the relative rarity of this presentation in the worldwide context. However, there is a possibility that patients who did not proceed to laparotomy or laparoscopy may have been presented later to another institution, thereby lessening the rate. Moreover, it is likely that the rate of negative surgical exploration in patients with GIP is lower than other areas with incomparable standards of care as well as the availability of CT. A further limitation is that CRP, PCT and D-dimer were not analyzed, which are regarded as infection index (30-32) and might be significant predictors of N-GIP. At last, as the number of individuals in GIP group was extremely different from that in N-GIP group, robust identification of predictors for N-GIP is limited by the sample size of patients who had negative surgical exploration. Thus, future studies with more universal laboratory testing and larger sample size are needed to verify and generalize our findings.

Despite these limitations, the present study had many strengths. It is the largest retrospective study evaluating the rate of negative surgical exploration in patients with 
suspected GIP. Second, the data span an interval of 11 years, longer than in most previous investigations. Finally, the model developed had high predictive value and has good generalizability to other patients undergoing laparotomy and laparoscopy for GIP.

\section{Conclusions}

The overall rate of negative surgical exploration in patients with suspected GIP was 3.1\% with no significant decrease over time. Applicating a multivariable logistic regression, we can easily identify predictive factors of N-GIP and help surgeons select a small portion of patients suspected of having GIP to minimize unnecessary emergency surgery, provide a unique opportunity for conservative management in stable patients, and expedite appropriate care.

\section{Acknowledgments}

We thank Dr. Yihong Sun for his permission to undertake the study and for guidance and support.

Funding: None.

\section{Footnote}

Reporting Checklist: The authors have completed the STROBE reporting checklist. Available at http://dx.doi. org/10.21037/atm-20-8158

Data Sharing Statement: Available at http://dx.doi. org/10.21037/atm-20-8158

Peer Review File: Available at http://dx.doi.org/10.21037/ atm-20-8158

Conflicts of Interest: All authors have completed the ICMJE uniform disclosure form (available at http://dx.doi. org/10.21037/atm-20-8158). The authors have no conflicts of interest to declare.

Ethical Statement: The authors are accountable for all aspects of the work in ensuring that questions related to the accuracy or integrity of any part of the work are appropriately investigated and resolved. The study was conducted in accordance with the Declaration of Helsinki (as revised in 2013). The study was approved by ethics board of Zhongshan Hospital, Fudan University (No.: B2021-116) and individual consent for this retrospective analysis was waived.

Open Access Statement: This is an Open Access article distributed in accordance with the Creative Commons Attribution-NonCommercial-NoDerivs 4.0 International License (CC BY-NC-ND 4.0), which permits the noncommercial replication and distribution of the article with the strict proviso that no changes or edits are made and the original work is properly cited (including links to both the formal publication through the relevant DOI and the license). See: https://creativecommons.org/licenses/by-nc-nd/4.0/.

\section{References}

1. McCaig LF, Burt CW. National Hospital Ambulatory Medical Care Survey: 2002 emergency department summary. Adv Data 2004;18:1-34.

2. Langell JT, Mulvihill SJ. Gastrointestinal perforation and the acute abdomen. Med Clin North Am 2008;92:599-625.

3. Del Gaizo AJ, Lall C, Allen BC, et al. From esophagus to rectum: a comprehensive review of alimentary tract perforations at computed tomography. Abdom Imaging 2014;39:802-23.

4. Dellinger RP, Carlet JM, Masur H, et al. Surviving Sepsis Campaign guidelines for management of severe sepsis and septic shock. Intensive Care Med 2004;30:536-55.

5. Ordoñez CA, Puyana JC. Management of peritonitis in the critically ill patient. Surg Clin North Am 2006;86:1323-49.

6. Hapani S, Chu D, Wu S. Risk of gastrointestinal perforation in patients with cancer treated with bevacizumab: a meta-analysis. Lancet Oncol 2009;10:559-68.

7. Xie F, Yun H, Bernatsky S, et al. Brief Report: Risk of Gastrointestinal Perforation Among Rheumatoid Arthritis Patients Receiving Tofacitinib, Tocilizumab, or Other Biologic Treatments. Arthritis Rheumatol 2016;68:2612-7.

8. Závada J, Lunt M, Davies R, et al. The risk of gastrointestinal perforations in patients with rheumatoid arthritis treated with anti-TNF therapy: results from the BSRBR-RA. Ann Rheum Dis 2014;73:252-5.

9. Søreide K, Thorsen K, Harrison EM, et al. Perforated peptic ulcer. Lancet 2015;386:1288-98.

10. Holmer C, Mallmann CA, Musch MA, et al. Surgical Management of Iatrogenic Perforation of the Gastrointestinal Tract: 15 Years of Experience in a Single Center. World J Surg 2017;41:1961-5.

11. Ahn SH, Mayo-Smith WW, Murphy BL, et al. Acute 
nontraumatic abdominal pain in adult patients: abdominal radiography compared with CT evaluation. Radiology 2002;225:159-64.

12. Tang A, Huddleston P, Attaluri P, et al. Clinical cases of nonsurgical pneumoperitoneum: categorizing the disease and treatment options. Am Surg 2015;81:E206-8.

13. Johnson EK, Choi YU, Jarrard SW, et al. Pneumoperitoneum after rough sexual intercourse. Am Surg 2002;68:430-3.

14. Mularski RA, Ciccolo ML, Rappaport WD. Nonsurgical causes of pneumoperitoneum. West J Med 1999;170:41-6.

15. Rowe NM, Kahn FB, Acinapura AJ, et al. Nonsurgical pneumoperitoneum: a case report and a review. Am Surg 1998;64:313-22.

16. Sambursky JA, Kumar S, Orban M, et al. Non-surgical Pneumoperitoneum in the Setting of Gram-negative Sepsis. Cureus 2018;10:e2493.

17. İflazoğlu N, Gokce ON, Kivrak MM, et al. Spontaneous idiopathic pneumoperitoneum with acute abdomen. Turkish Journal of Surgery 2013;31:110-2.

18. Roh JJ, Thompson JS, Harned RK, et al. Value of pneumoperitoneum in the diagnosis of visceral perforation. Am J Surg 1983;146:830-3.

19. Winek TG, Mosely HS, Grout G, et al. Pneumoperitoneum and its association with ruptured abdominal viscus. Arch Surg 1988;123:709-12.

20. Mularski RA, Sippel JM, Osborne ML. Pneumoperitoneum: A review of nonsurgical causes. Crit Care Med 2000;28:2638-44.

21. Hoover EL, Cole GD, Mitchell LS, et al. Avoiding laparotomy in nonsurgical pneumoperitoneum. Am J Surg 1992;164:99-103.

22. Eisenbach C, Blaker H, Stremmel W, et al. Pneumoperitoneum following endoscopic mucosal resection without perforation of the colon. Endoscopy

Cite this article as: Liu X, Sheng W, Gong Y, Gao W, Zhang B. Negative surgical exploration in suspected gastrointestinal perforation: trend, preoperative predictors, and etiologies. Ann Transl Med 2021;9(10):832. doi: 10.21037/atm-20-8158
2008;40 Suppl 2:E64-5.

23. Alassaf M. Recurring spontaneous aseptic pneumoperitoneum presenting secondary to an unrelated chief complaint: A case report. Int J Surg Case Rep 2015;7C:96-8.

24. Napolitano L, Waku M, Maggi G, et al. Cystic intestinal pneumatosis of the small gut: Etiopathogenesis and review of literature. Ann Ital Chir 2018;7:S2239253X18028153.

25. Fultz PJ, Skucas J, Weiss SL. CT in upper gastrointestinal tract perforations secondary to peptic ulcer disease. Gastrointest Radiol 1992;17:5-8.

26. Urban BA, Fishman EK. Tailored helical CT evaluation of acute abdomen. Radiographics 2000;20:725-49.

27. Omori H, Asahi H, Inoue Y, et al. Selective application of laparoscopic intervention in the management of isolated bowel rupture in blunt abdominal trauma. J Laparoendosc Adv Surg Tech A 2003;13:83-8.

28. An SB, Kim BC, Kim JY, et al. Results of Laparotomy and Laparoscopy for Perforated Colonic Diverticulitis. JSLS 2019;23:e2019.00007.

29. Branicki FJ. Abdominal emergencies: diagnostic and therapeutic laparoscopy. Surg Infect (Larchmt) 2002;3:269-82.

30. Schlattmann P, Brunkhorst FM. Procalcitonin as a diagnostic marker for sepsis. Lancet Infect Dis 2014;14:189.

31. Hu L, Shi Q, Shi M, et al. Diagnostic Value of PCT and CRP for Detecting Serious Bacterial Infections in Patients With Fever of Unknown Origin: A Systematic Review and Meta-analysis. Appl Immunohistochem Mol Morphol 2017;25:e61-9.

32. Mikuła T, Sapula M, Jablonska J, et al. Significance of Heparin-Binding Protein and D-dimers in the Early Diagnosis of Spontaneous Bacterial Peritonitis. Mediators Inflamm 2018;2018:1969108. 